\title{
A Novel Analyzing Method to Coal Mine Image Restoration
}

\author{
Changchun Shang \\ Xi'an University of Science and Technology \\ Engineering Training Center \\ Xi'an, China
}

\begin{abstract}
In order to solve the phenomena that harsh coal mine environment will lead to coal mine monitoring image degradation, a K-fold Cross-Validation image restoration algorithm BP neural network was proposed. Firstly, the images will be blurred by Gaussian white noise. Then, the blurred image and original image match "training pairs". When the training error and validation error is equal, stop the network training, select the training error and test error are smaller as the optimal model. Finally, bring the blurred image to the restoration model and image processing. Experiment shows that the K-Fold Cross-Validation BP neural network model for image restoration of generalization performance and fitting precision both meet the requirements.
\end{abstract}

Keywords-Image restorations; K-fold Cross-Validation;BP neural network; Coal mine.

\section{INTRODUCTION}

At present, the coal mine use the industrial technology to ensure the production security and high-efficiency. But mine usually has insufficient light, low intensity of illumination, dust, in getting image, and other various reasons which make image quality drop and image degradation. Therefore, in the coal mine field of image processing, image restoration processing is becoming a focus of attention [1].The purpose of image restoration is based on appropriate optimization criterion to get clear images of and as close as possible to the original image, such as image processing, pattern recognition, machine vision, which has been the basis of widely research [2]. The classic image restoration methods are mostly through the way of deconvolution, and in the process of deconvolution, however, if pathological problems or multiple solution problem exist, which is difficult to solve. In addition, the image of qualitative model is not allowed in many cases is unknown or estimate, makes the classic image restoration methods [3] is difficult due to lack of prior knowledge. Recovery methods are divided into the blind restoration algorithm and blind restoration algorithm [4].In actual application, A blind restoration algorithm is difficult to determine the point spread function in advance; on the other hand, Blind restoration algorithm need to use some known incomplete information, large amount of calculation, so the speed is too slow.

In recent years, image restoration algorithm based on BP neural network has become one of the hot topics in the field of image restoration [5-6]. Since BP neural network has a strong learning and generalization capabilities it has wide range field in image processing applications. While the BP neural network also has some shortcomings, such as a large amount of computation, slow convergence and easy to fall into local minima and other defects. In this paper, the author proposed a combination of image restoration algorithm BP K-Fold Cross-Validation with a sample images blurred and artificial neural network forecasting model. For a given, only a limited learning task training data it will achieve a good balance of accuracy and machine specific training data from the learning ability (machine without error to learn any training data). Specifically, the use of K-Fold Cross-Validation, meanwhile change the neural network training termination conditions, and then the trained BP neural network model was optimal selection, which finally will get a good generalization performance optimization model of progressive image restoration.

\section{K-FOLD CROSS-VALIDATION AND BP NEURAL NETWORK}

BP neural network is a kind of multi-stage feedforward network, to have a mentor learning method, establishing of highly nonlinear mapping relationship between input and output.

BP network is composed of input layer, output layer and hidden layer, each layer is composed of a number of neurons, by weight connection between adjacent layers.

Neural network generalization ability is refers to the learning of neural network model to test sample or samples to make the right response ability. In general, the neural network train network by training set and validate network by validation set; so a network model should meet a requirement when the training error is less than the set value in advance to stop training,. But, in the actual process of neural network training, small training error does not always lead to good prediction effect; Specifically, for limited sample of learning ability is strong and enough to remember every sample, the experience can quickly converge to a small or even zero risk, but there is no guarantee that it can give a good response to verify the concentration of sample; Moreover, at the same time, the validation error is not conform to the proportional relationship between the training error, so the network will appear "over fitting " phenomenon, as shown in Figure 1. 


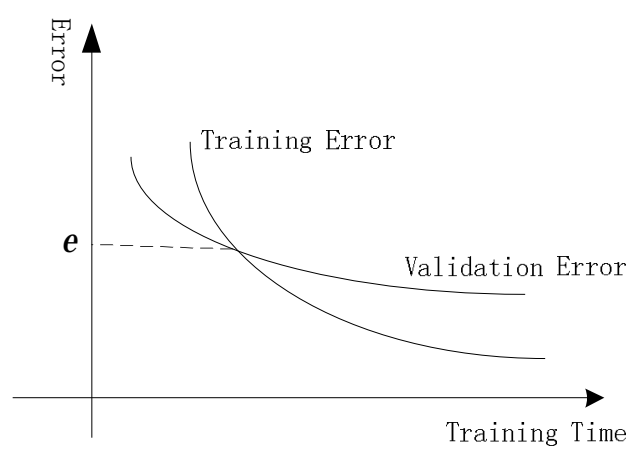

Figure.1 The "Over Fitting" phenomenon of the neural network

\section{A. K-Fold Cross-Validation principle}

Using cross validation method to study the data sample is divided into two parts [7]: training data image and validate image samples. In order to get a better effect, regardless of the training sample or validation samples as far as possible to participate in the study. In this paper, the recovery image sample Cross Validation method according to $\mathrm{K}$ ( $\mathrm{K}$ - a fold Cross-Validation) evenly divided into $K$ copies. Randomly select the $K-1$ as a training set, the remaining 1 as a validation set. Therefore,

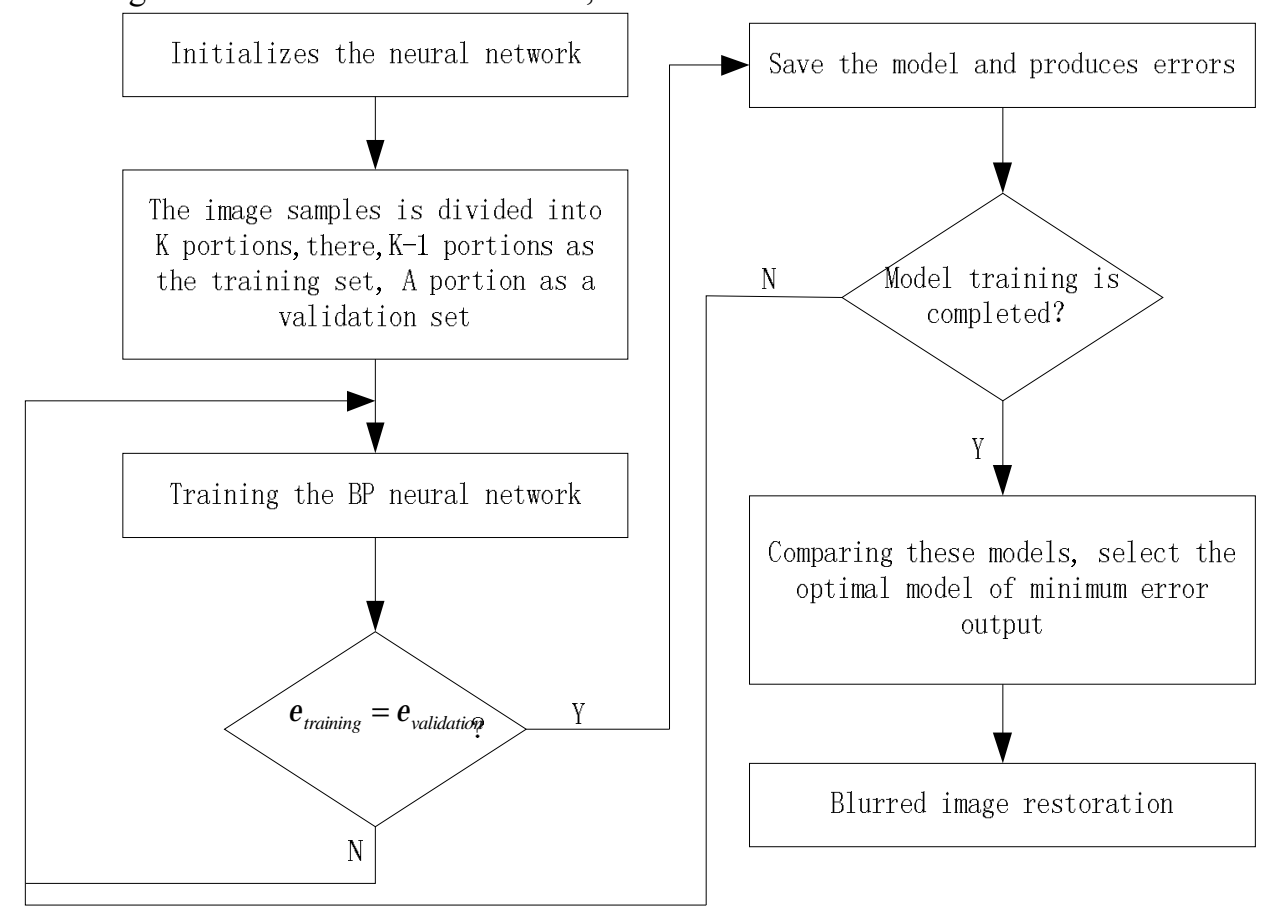

Figure.2: The Image Restoration Flowchart based on K-fold Cross-Validation BP Network

Initializes the network weights, the network transmission function are selected as the sigmoid function. The function is shown as equation (1).

$$
f(x)=1 /[1+\exp (-x)]
$$

Step 1: Blur the original image and preprocess it, put the Gaussian blur grayscale image as input, put original gray image as output. input. there is a total of $\mathrm{K}$ method, which can get $\mathrm{K}$ model,, Finally select optimal model by smaller training error and test error .

As shown in figure 1, for the current training sample and verified sample, with the decrease of the training error, validation error decreases and then increases, and the generalization capability of feedforward neural network is gradually reduced. In order to prevent network overtraining and generalization ability is reduced, when training error is equal to the validation error, stop training. At this time, meet the conditions to get the best network model generalization performance. At the same time, the model has an error.

Through the above method, the original $\mathrm{K}$ samples combined random at a time, eventually produce $\mathrm{K}$ model is composed of a number of samples training; Each model has its corresponding error, and their generalization performance for each model are better samples. Then select the minimum error from the $\mathrm{K}$ model, this model is not only high fitting precision, but also the strong generalization ability and the corresponding model stability is better.

\section{B. The Implementation of the Algorithm}

Using the neural network gradual optimization algorithm process is shown in Figure 2.

Step 3: With K - fold Cross-Validation method to the sample data is divided into $\mathrm{K}, \mathrm{K}-1$ as a training set, 1 as a Validation set.

Step 4: Using the neural network training, at the time, the current training stopped, save training model and error. If the training error and validation are not equal, we continue the training. 
Step 5: When K model all after the training, the comparison of model selection of K model, select the optimal model of minimum error output.

Step 6: Put the blurred images into the optimal model for image restoration.

\section{EXAMPLE USE}

According to the principle of the optimization algorithm, build image restoration $\mathrm{K}$ fold cross-validation BP neural network model.

(1) Pre-process the sample images and get the corresponding gray images as input, shown as Figure.3.
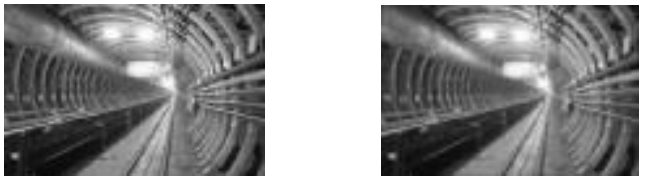

(a) $1^{\#}$ Original Image

(b) $1^{\#}$ Image with Gaussian Blurred
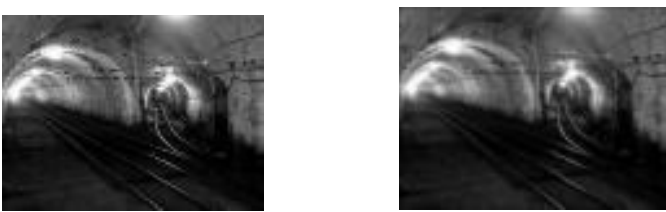

(c) $2^{\#}$ Original Image

(d) $2^{\#}$ Image with Gaussian Blurred
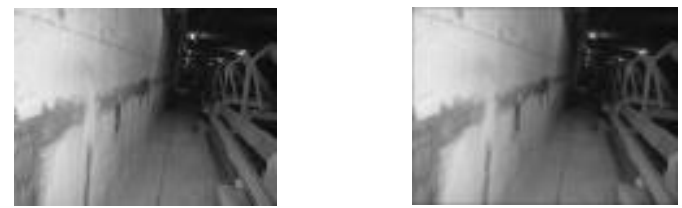

(e) $3^{\#}$ Original Image

(f) $3^{\#}$ Image with Gaussian Blurred Figure. 3 Part of the training samples

(2) According to the K-fold Cross-Validation, 15 images can be divided into 5 groups randomly, each selected 4 groups as the training data, 1 set as validation data and so on.

(3) Set the parameters of neural network training; determine the neuron output for corrosion rate.

(4) Simulation data are normalized, if the training error $=$ validation error, or the training error and validation error close (|training error - validation error| $\leq 0.05$ ), the current training is terminated, save the model, and record the training error. So, when all the combination after the training sample data, produce five models, get five training error, selects the smallest error corresponding training model which is the optimal model.

After training of neural network to get five model, finally from five training error as shown in table 1 (table "----" represents when training to set times, |training error - validation error $>0.05)$.

TABLE I. MODEL TRAINING ERROR

\begin{tabular}{|l|c|c|c|l|l|}
\hline Model & 1 & 2 & 3 & 4 & 5 \\
\hline $\begin{array}{l}\text { Training } \\
\text { Error }\end{array}$ & 0.1014 & - & 0.0955 & 0.1165 & 0.1200 \\
\hline
\end{tabular}

By comparing the nine model training error in Table 1, the third model has minimum error; model training is $\mathrm{K}$ fold to choose the optimal model. The weight of the training model is shown as figure 4. Model training error is shown in Figure.5.

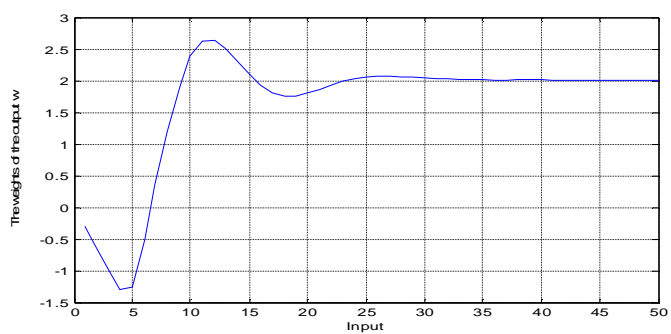

Figure.4:The Weight of Training Model

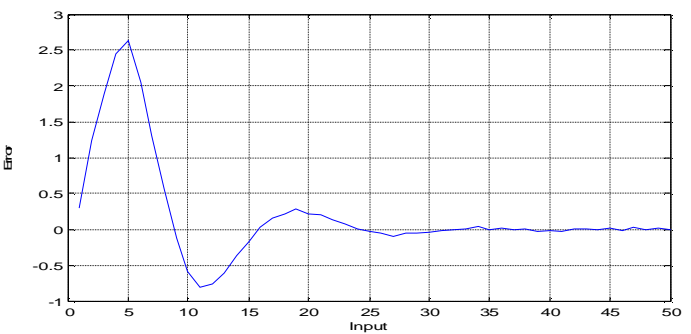

Figure.5: Model Training Error

It is shown in Figure.4 and Figure.5. A weight shock attenuation, and gradually converge to two nearby, the overall convergence effect is good.

This paper select coal mine picture and test the above algorithms. In figure 6 , (a) is the original image, as an idealized without noise image;(b) is blurred image, is no noise of idealized images after Gaussian blur image; (c) is the denatured image by the traditional BP neural network $(\mathrm{d})$ is the denatured image by $\mathrm{K}$-fold crossvalidation BP network. Actually, an important factor of evaluating image quality is subjective visual effect [8], from the point of subjective visual figure 3, the images of the BP neural network restoration, has shortcomings like the presence of large amounts of noise, edge blur, which seriously affect the image quality. Images of K-fold crossvalidation BP network restoration, not only improved the BP algorithm to deal with the deficiency of the image, but also in the aspect of image target signs keep effect is good and image details are improved greatly. 


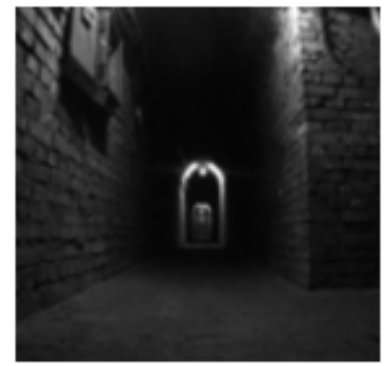

(a)Original Image

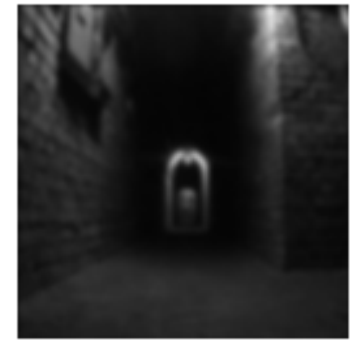

(b)Image with Gaussian Blurred

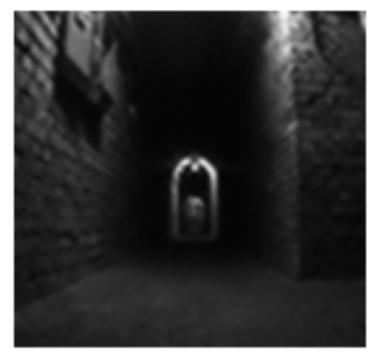

(c)The Image base on BP Network

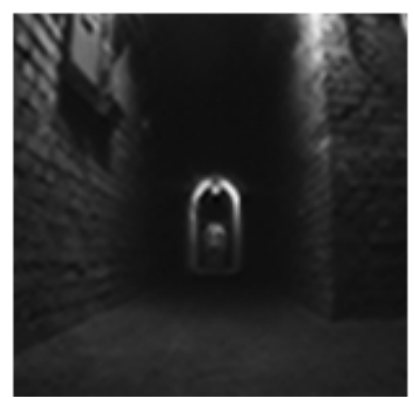

(d)the Image base on our Algorithm Figure.6: Image restoration test pattern

As the research in this paper, the performance of the proposed model, this article takes the SSIM (structural similarity index) and PSNR (Peak Signal to Noise Ration) performance analysis [9].

Table 2 shows that the strategy based on the basis of BP neural network image Restoration can reluctantly realize image restoration. But the strategy based on the Kfold Cross-Validation BP neural network image can accurately realize image restoration in the criteria of SSIM and PSNR.
TABLE II.

PERFORMANCE ANALYSIS

\begin{tabular}{|c|c|c|c|c|}
\hline $\begin{array}{c}\text { Metho } \\
\text { ds }\end{array}$ & \multicolumn{2}{|c|}{$\begin{array}{c}\text { Traditional BP } \\
\text { Neural Network }\end{array}$} & \multicolumn{2}{c|}{$\begin{array}{c}\text { K-Fold Cross- } \\
\text { Validation BP } \\
\text { Neural Network }\end{array}$} \\
\hline Model & $\begin{array}{c}\text { Training } \\
\text { Model }\end{array}$ & $\begin{array}{c}\text { Validatio } \\
\text { n Model }\end{array}$ & $\begin{array}{c}\text { Training } \\
\text { Model }\end{array}$ & $\begin{array}{c}\text { Validatio } \\
\text { n Model }\end{array}$ \\
\hline SSIM & 0.9755 & 0.9592 & 0.9852 & 0.9631 \\
\hline PSNR & 32.5593 & 34.5287 & 32.6935 & 34.8862 \\
\hline
\end{tabular}

\section{CONCLUSIONS}

This paper proposes a model based on K-Fold CrossValidation BP neural network used in image restoration of Coal mine detection robot vision system. In view of the limited study sample data, using cross validation method for training for many times, get multiple model; As the same time, change neural network training termination conditions train the selection model, and then choose the optimal model of multiple model for image restoration, finally, get good stability and strong generalization ability of the optimal model. The simulation results show the model has a high and stable performance.

\section{REFERENCES}

[1] Ying Dongjie,Li Wenjie. Analysis of Enhancement Algorithms of coal mine monitoring image and its realization[J]. Industry and Mine Automation, 2012, 8(8):55-58.

[2] GUO Pei, HE Xiaohai,TAO Qingchuan, et al. Restoration method of PSO-BP neural network based on HDSG[J]. Computer Engineering and Applications, 2012, 48(2):156-159.

[3] Zhou Changying. Research on Image Segmentation Technology Based on Improved Fuzzy BP Neural Network[J]. Computer Simulation, 2011, 28(4): 287-290.

[4] Zhang Yinxue,Jia Zhenhong,Liu Zijian. Image filtering based on modified BP neural network and PSO [J]. Journal of Optoelectronics \& Laser, 2009, 20(3):104-109.

[5] Song Faxing,Gao Liuyang,Liu Dongsheng et al. A Method of Image Restoration Based on Particle Swarm Optimization for BP Neural Network[J]. Computer Technology and Development. 2014 24(6):149-153.

[6] Wang wan, Zhang Junwen, Lin Ming, et al. Life prediction of buried Gas pipeline based on the configuration of BP network model[J]. Modern Machinery, 2010(3):44-46.

[7] Lan H Witten,Eibe Frank.Data mining[M]. Mechanical Industry Press, Beijing (in Chinese), 2005:149-151.

[8] Zhang Ling,Zhang Yun,Yang Yimin. Color images restoration with multi-layer morphological ( MLM ) neural network [C] / Proc of 2003 international conference on machine learning and cybernetics. Xi'an,China,2003.

[9] Z. Wang, A. C. Bovik, H. R. Sheikh, and E. P. Simoncelli. Image quality assessment: From error visibility to structural similarity. IEEE Transaction on Image Processing, 2004, 13(4):600-612.

[10] Zhou Changying. Research on Image Segmentation Technology

[11] Based on Improved Fuzzy BP NeuralNetwork[J].The computer simulation.2011,28(4):287-290. 